MEDDELANDEN FRÅN SVENSKA HANDELSHÖGSKOLAN

SWEDISH SCHOOL OF ECONOMICS AND BUSINESS ADMINISTRATION

WORKING PAPERS

460

Luis H. R. Alvarez \& Rune Stenbacka

OPTIMAL RISK ADOPTION:

A REAL OPTIONS APPROACH 
Key words: Risk adoption, investment, real option

AMS: 90A09, 90A11, 62L15, 60G40, 60J60

JEL: O32, G30, D92, C61

(C) Swedish School of Economics and Business Administration, Luis H. R. Alvarez \& Rune Stenbacka

Distributor:

\section{Library}

Swedish School of Economics and Business Administration

P.O.Box 479

00101 Helsinki

Finland

Phone: +358-9-431 33 376, +358-9-431 33265

Fax: +358-9-43133425

E-mail:publ@shh.fi

http://www.shh.fi/link/bib/publications.htm

SHS intressebyrå IB (Oy Casa Security Ab), Helsingfors 2001

ISBN 951-555-697-X

ISSN 0357-4598 


\title{
Optimal Risk Adoption: A Real Options Approach
}

\author{
Luis H. R. Alvarez ${ }^{1}$ \\ Turku School of Economics and Business Administration \\ Department of Economics \\ Economic Mathematics and Statistics \\ FIN-20500 Turku \\ Finland \\ Rune Stenbacka ${ }^{2}$ \\ Swedish School of Economics and Business Administration \\ Department of Economics \\ P.O. Box 479 \\ FIN-00101 Helsinki \\ Finland
}

May 16, 2001

\begin{abstract}
This study develops a real options approach for analyzing the optimal risk adoption policy in an environment where the adoption means a switch from one stochastic flow representation into another. We establish that increased volatility needs not decelerate investment, as predicted by the standard literature on real options, once the underlying volatility of the state variable is made endogenous. We prove that for a decision maker with a convex (concave) objective function, increased post-adoption volatility increases (decreases) the expected cumulative present value of the post-adoption profit flow, which consequently decreases (increases) the option value of waiting and, therefore, accelerates (decelerates) current investment.
\end{abstract}

Keywords: Risk adoption, investment, real option.

AMS Subject Classification: 90A09, 90A11, 62L15, 60G40, 60J60.

JEL Subject Classification: O32, G30, D92, C61.

\footnotetext{
${ }^{1}$ e-mail: luis.alvarez@tukkk.fi

2 e-mail: rune.stenbacka@shh.fi
} 


\section{Introduction}

For a firm operating in an uncertain environment the irreversible adoption of a new technology or the irreversible entry into a new market mean a switch from one stochastic profit flow representation into another. Within the framework of a real options approach such a regime switch is captured by a change in the stochastic differential equation characterizing the evolvement of the state variable. Risk adoptions in this general sense capture a large spectrum of economic applications. It might be associated with a technological upgrading of an uncertain production technology. It could also cover a firm's entry into new markets so that the firm's risk exposure would change. Further examples of similar types of regime shifts can be found within financial markets where announcements (cf. Grenadier (1999)) often generate shifts in the return pattern due to the associated information disclosure or where observations of the trading patterns of an investor might impact on the stochastic process determining the asset return.

Traditional neoclassical investment theory (for example, Abel $(1983,1984,1985)$ and Hartman $(1972,1973))$ predicted a positive relationship between volatility and investment in the sense that increased stochastic return fluctuations would speed up investment by increasing the expected cumulative present value of future marginal productivity improvements. In contrast to such a positive relationship between volatility and investment the real options approach, as shaped by influential contributions such as Pindyck $(1988,1991,1993)$, Dixit $(1989,1992)$ or Dixit and Pindyck (1994), seems to imply a negative relationship between uncertainty and investment. For example, Dixit and Pindyck (1994) (p. 373) conclude that:

"a larger $\sigma$ (standard deviation of demand) means a lower long-run average growth rate of the capital stock, and thus less investment on average."

In a similar spirit Caballero (1991) and Lee and Shin (2000) develop two-period models where firms start off with no capital and where the real option effect (the ability to postpone the exercise of the investment opportunity) of uncertainty acts to reduce investment in the first period.

The real options approach characterizes the comparative statics properties of how the optimal timing of an irreversible investment behaves as a function of the diffusion coefficient of the underlying stochastic process and this relationship is typically given the interpretation of a negative relationship between uncertainty and investment. In the present paper we actually address this issue in a more general framework by asking: Under which circumstances will a firm have an incentive to pay an irreversible investment cost in order to induce a switch from one stochastic process into another? Our analysis offers a characterization of an optimal risk adoption policy as an interplay between three factors: (i) the incorporated risks as delineated by the relative magnitudes of the risk measures (the volatilities) of the two stochastic processes, (ii) the irreversible adoption (investment) cost and (iii) the functional relationship between the state variable and the profit (objective) function of the firm (the decision maker). Under which circumstances will a firm have incentives to switch to a more risky technology or enter a more risky market? How do these incentives for increased (decreased) risk exposure interact with the characteristics of the profit function whereby the state variable is mapped into the objective function of the decision maker?

The feature whereby the optimal risk adoption policy is generated through the interplay between the stochastic fluctuations incorporated in the projects available and the functional relationship between the state variable and the objective function of the decision maker in charge of the firm's risk taking is a remarkable and important conclusion. It is a crucial result with implications for the consequences of delegation within a principal-agent perspective of the firm. Namely, our characterization demonstrates how the principal's selection of incentive contract for the agent will affect the firm's risk taking and how the risk implications of this 
delegation issue interact with the underlying risk measures of the spectrum of investment projects available to the agent.

In this article, we will show, contrary to the standard literature on real options, that increased volatility needs not decelerate investment. In fact, we characterize circumstances under which the standard neoclassical hypotheses of a positive relationship between risk and investment can be supported even when accounting for the real option value of waiting to make investment commitments. In this respect our study unifies the seemingly contradicting predictions of the two main approaches to investment theory, the neoclassical approach and the real option approach. More precisely, we exhibit that the determination of the optimal risk adoption can be viewed as a trade-off between the value of flexibility associated with waiting and the form of the functional relationship between the state variable and the profit flow. We find that for a decision maker with a convex (concave) objective function, increased post-adoption volatility increases (decreases) the expected cumulative present value of the post-adoption profit flow, which consequently decreases (increases) the option value of waiting and, therefore, accelerates (decelerates) current investment. Analogous conclusions are reached with respect to the impact of pre-adoption volatility on investment.

Our analysis offers interesting perspectives on the traditional textbook versions of the Capital Asset Pricing Model (CAPM). CAPM predicts a positive equilibrium relationship between expect return and risk in the sense of specifying an equilibrium price at which an investor is willing to accept an increase in risk. In contrast, our real options approach offer a characterization of circumstances under which an investor would be willing to pay an irreversible adoption cost in order to switch to a stochastic return process with a higher, not lower, incorporated risk. Of course, under such circumstances the equilibrium relationship between risk and expected return would drastically change with strong implications for a virtual market of assets represented by irreversible investment projects.

Our study proceeds as follows. In section two we characterize the optimal risk adoption as the interplay between the real option effect and the Jensen's inequality effect for a coupled geometric Brownian motion. Subsequently section three extend the coupled process to more general stochastic dynamics. Finally, section four offers some concluding comments.

\section{Optimal Risk Adoption}

Assume that the firm's ongoing business yields a stochastic return. Assume also that this firm faces an business development opportunity, for example an investment opportunity which fundamentally changes the stochastic fluctuations associated with the firm's profit flow. In order to characterize the optimal timing of when to adopt the new investment opportunity, we initially consider the optimal stopping problem

$$
V(x)=\sup _{\tau} \mathbb{E}_{x}\left[\int_{0}^{\infty} e^{-r s} f(X(s)) d s-e^{-r \tau} c\right],
$$

where $r>0$ denotes the risk-free rate of return, $c$ is a known adoption cost (exercise price), $f: \mathbb{R}_{+} \mapsto \mathbb{R}_{+}$is a known mapping measuring the net revenue flow of operation, $\{X(t) ; t \geq 0\}$ is a linear diffusion defined on a complete filtered probability space $\left(\Omega, \mathbb{P},\left\{\mathcal{F}_{t}\right\}_{t \geq 0}, \mathcal{F}\right)$, and $\tau$ is an arbitrary $\mathcal{F}_{t}$-stopping time. In accordance with most applications in economics and finance, we assume that $\{X(t) ; t \geq 0\}$ evolves on $\mathbb{R}_{+}$according to the stochastic differential equation

$$
d X(t)=\mu X(t) d t+\sigma(t) X(t) d W(t), \quad X(0):=x,
$$

where

$$
\sigma(t)= \begin{cases}\sigma_{1}, & t<\tau \\ \sigma_{2}, & t \geq \tau\end{cases}
$$


and $\mu \in \mathbb{R}$ as well as $\sigma_{1} \neq \sigma_{2} \in \mathbb{R}_{+}$are exogenously determined constants. In other words, we assume that $X(t)$ constitutes a coupled diffusion process

$$
X(t)= \begin{cases}X_{1}(t), & t<\tau \\ X_{2}(t), & t \geq \tau\end{cases}
$$

where $\tau$ is the coupling date (which in the present case is an endogenously determined $\mathcal{F}_{t^{-}}$ stopping time which has to be solved from the optimal adoption problem (1)),

$$
d X_{i}(t)=\mu X(t) d t+\sigma_{i} X(t) d W(t)
$$

$X_{1}(0)=x$, and $X_{1}(\tau-)=X_{2}(\tau+)$. Thus, the irreversible adoption decision changes the diffusion coefficient in reflection of a switch in the generated riskiness of the profit flow, while the drift remains constant. Consequently, contrary to the earlier literature we analyze risk adoption not relative to a benchmark characterized by no uncertainty, but rather we focus on regime switches from a given degree of uncertainty into another. However, it is worth emphasizing that our analysis also incorporates as a special case the traditional benchmark situation of an incumbent (updated) technology with no uncertainty. As usually, we denote as $\mathcal{A}_{i}$ the differential operator

$$
\mathcal{A}_{i}=\frac{1}{2} \sigma_{i}^{2} x^{2} \frac{d^{2}}{d x^{2}}+\mu x \frac{d}{d x},
$$

representing the infinitesimal generator of the diffusion $X_{i}, i=1,2$.

We now denote as $\mathcal{L}_{i}^{1}(\mathbb{P})$ the class of measurable mappings satisfying the absolute integrability condition (which is known as the absence of speculative bubbles condition)

$$
\mathbb{E}_{x} \int_{0}^{\infty} e^{-r s}\left|f\left(X_{i}(s)\right)\right| d s<\infty, i=1,2,
$$

and define for $f \in \mathcal{L}_{i}^{1}(\mathbb{P})$ the functionals $\left(R_{r} f\right)_{i}: \mathbb{R}_{+} \mapsto \mathbb{R}$ as

$$
\left(R_{r} f\right)_{i}(x)=\mathbb{E}_{x} \int_{0}^{\infty} e^{-r s} f\left(X_{i}(s)\right) d s<\infty, i=1,2 .
$$

In other words, $\left(R_{r} f\right)_{i}(x)$ can be interpreted as the expected cumulative present value of the revenue flow $f(x)$ in the presence of the price process $X_{i}(t)$ and $\mathcal{L}_{i}^{1}(\mathbb{P})$ can be interpreted as the class of revenue flows for which the expected cumulative present value from the present up to an arbitrarily distant future is well-defined.

It is clear that if $f \in \mathcal{L}_{1}^{1}(\mathbb{P}) \cap \mathcal{L}_{2}^{1}(\mathbb{P})$, then the strong Markov property of diffusions implies that problem (1) can be rewritten as

$$
V(x)=\left(R_{r} f\right)_{1}(x)+\sup _{\tau} \mathbb{E}_{x}\left[e^{-r \tau}\left(\left(R_{r} f\right)_{2}\left(X_{1}(\tau)\right)-\left(R_{r} f\right)_{1}\left(X_{1}(\tau)\right)-c\right)\right] .
$$

The first term in the right hand side of (8) denotes the payoff associated with the incumbent technology, while the second term can be given an interpretation as an exercise or adoption premium. An important characterization of the expected cumulative present values $\left(R_{r} f\right)_{i}(x)$ needed later in this study is now presented in

Lemma 1. (A) Assume that $f(x)$ is convex, and that $f \in \mathcal{L}_{i}^{1}(\mathbb{P})$. Then, $\left(R_{r} f\right)_{i}(x)$ is convex on $\mathbb{R}_{+}$.

(B) Assume that $f(x)$ is concave, and that $f \in \mathcal{L}_{i}^{1}(\mathbb{P})$. Then, $\left(R_{r} f\right)_{i}(x)$ is concave on $\mathbb{R}_{+}$. 
Proof. (A) Denote now as $X_{i}^{x}(t)$ the solution of the stochastic differential equation (5) when initiated from the state $x \in \mathbb{R}_{+}$, that is, let

$$
X_{i}^{x}(t)=x e^{\left(\mu-\sigma_{i}^{2} / 2\right) t+\sigma_{i} W(t)} .
$$

If $x, y \in \mathbb{R}_{+}, \lambda \in[0,1]$, and $\hat{x}=\lambda x+(1-\lambda) y$, we find that

$$
X_{i}^{\hat{x}}(t)=\lambda X_{i}^{x}(t)+(1-\lambda) X_{i}^{y}(t)
$$

a.s. for all $t \geq 0$. Thus, if $f \in \mathcal{L}_{i}^{1}(\mathbb{P})$ is convex we find that

$$
\begin{aligned}
\left(R_{r} f\right)_{i}(\hat{x}) & =\mathbb{E} \int_{0}^{\infty} e^{-r s} f\left(X_{i}^{\hat{x}}(s)\right) d s=\mathbb{E} \int_{0}^{\infty} e^{-r s} f\left(\lambda X_{i}^{x}(s)+(1-\lambda) X_{i}^{y}(s)\right) d s \\
& \leq \mathbb{E} \int_{0}^{\infty} e^{-r s}\left[\lambda f\left(X_{i}^{x}(s)\right)+(1-\lambda) f\left(X_{i}^{y}(s)\right)\right] d s \\
& =\lambda\left(R_{r} f\right)_{i}(x)+(1-\lambda)\left(R_{r} f\right)_{i}(y)
\end{aligned}
$$

proving the alleged convexity of $\left(R_{r} f\right)_{i}(x)$ on $\mathbb{R}_{+}$. The proof of part (B) is completely analogous.

Lemma 1 demonstrates the intuitively clear result that the convexity or concavity of the revenue flow $f(x)$ is preserved when evaluating the expected cumulative present values $\left(R_{r} f\right)_{i}(x)$. A key consequence of this lemma is now presented in

Lemma 2. (A) Assume that $\sigma_{2} \geq \sigma_{1}$, that $f(x)$ is convex, and that $f \in \mathcal{L}_{2}^{1}(\mathbb{P})$. Then, $\left(R_{r} f\right)_{1}(x) \leq\left(R_{r} f\right)_{2}(x)$ for all $x \in \mathbb{R}_{+}$.

(B) Assume that $\sigma_{2} \geq \sigma_{1}$, that $f(x)$ is concave, and that $f \in \mathcal{L}_{1}^{1}(\mathbb{P})$. Then, $\left(R_{r} f\right)_{1}(x) \geq$ $\left(R_{r} f\right)_{2}(x)$ for all $x \in \mathbb{R}_{+}$.

Proof. (A) Assume that $\sigma_{2} \geq \sigma_{1}$, that $f(x)$ is convex, and that $f \in \mathcal{L}_{2}^{1}(\mathbb{P})$. It is a well-known result from the classical theory of diffusions that since the boundaries of the state-space of $X_{2}(t)$ are natural, $\left(R_{r} f\right)_{2}(x)$ constitutes the unique bounded solution of the ordinary second order differential equation $\left(\left(\mathcal{A}_{2}-r\right) u\right)(x)+f(x)=0$ (cf. Mandl (1968), p. 34). Consequently, we observe that for all $x \in \mathbb{R}_{+}$

$$
\left(\left(\mathcal{A}_{1}-r\right)\left(R_{r} f\right)_{2}\right)(x)+f(x)=\left(\left(\mathcal{A}_{1}-\mathcal{A}_{2}\right)\left(R_{r} f\right)_{2}\right)(x)=\frac{1}{2}\left(\sigma_{1}-\sigma_{2}\right) x^{2}\left(R_{r} f\right)_{2}^{\prime \prime}(x) \leq 0
$$

by the convexity of $f$ and the inequality $\sigma_{2} \geq \sigma_{1}$. Therefore, if $x \in \mathbb{R}_{+}$then

$$
\begin{aligned}
0 \leq \mathbb{E}_{x}\left[e^{-r T_{n}}\left(R_{r} f\right)_{2}\left(X_{1}\left(T_{n}\right)\right)\right] & =\left(R_{r} f\right)_{2}(x)+\mathbb{E}_{x} \int_{0}^{T_{n}} e^{-r s}\left(\left(\mathcal{A}_{1}-r\right)\left(R_{r} f\right)_{2}\right)\left(X_{1}(s)\right) d s \\
& \leq\left(R_{r} f\right)_{2}(x)-\mathbb{E}_{x} \int_{0}^{T_{n}} e^{-r s} f\left(X_{1}(s)\right) d s
\end{aligned}
$$

where $n \geq 2$ and $T_{n}=\min \left(n, \inf \left\{t \geq 0: X_{1}(t) \notin(1 / n, n)\right\}\right)$ is an almost surely finite stopping time. Reordering terms then yields that

$$
\mathbb{E}_{x} \int_{0}^{T_{n}} e^{-r s} f\left(X_{1}(s)\right) d s \leq\left(R_{r} f\right)_{2}(x) .
$$

Letting $n$ tend to infinity and invoking monotonic convergence then yields that $\left(R_{r} f\right)_{1}(x) \leq$ $\left(R_{r} f\right)_{2}(x)$ for all $x \in \mathbb{R}_{+}$. The proof of part (B) is completely analogous. 
Lemma 2 demonstrates the important result that given the boundedness of the considered functionals, increased volatility increases the expected cumulative present value of the future cash flows when evaluated from the point of view of a decision maker with an objective function that is convex in the state variable. The opposite argument is valid whenever the decision maker is endowed with a concave objective function. The key consequence of these results is now presented in

Proposition 1. (A) Assume that $f \in \mathcal{L}_{1}^{1}(\mathbb{P}) \cap \mathcal{L}_{2}^{1}(\mathbb{P})$ is convex and $\sigma_{1}<\sigma_{2}$. Then, increased post-exercise volatility $\sigma_{2}$ increases the value $V(x)$.

(B) Assume that $f \in \mathcal{L}_{1}^{1}(\mathbb{P}) \cap \mathcal{L}_{2}^{1}(\mathbb{P})$ is concave and $\sigma_{1}>\sigma_{2}$. Then, increased post-exercise volatility $\sigma_{2}$ decreases the value $V(x)$.

(C) Assume that $f \in \mathcal{L}_{1}^{1}(\mathbb{P})$ and that either $f(x)$ is convex and $\sigma_{2} \leq \sigma_{1}$, or that $f(x)$ is concave and $\sigma_{2} \geq \sigma_{1}$. Then, the option is never exercised (i.e. $\tau=\infty$ ) and $V(x)=$ $\left(R_{r} f\right)_{1}(x)$ for all $x \in \mathbb{R}_{+}$.

Proof. (A) As was demonstrated in Lemma 2, given our conditions increased post-exercise volatility increases the expected cumulative present value $\left(R_{r} f\right)_{2}(x)$. Since this is the only factor of (8) depending on post-exercise volatility $\sigma_{2}$ and expectation preserves ordering, we find that increased post-exercise volatility increases the value $V(x)$ as well. The proof of part (B) is completely analogous. (C) In light of Lemma 2, it is clear that if one of the conditions of our corollary is satisfied, then $\left(R_{r} f\right)_{2}(x)-\left(R_{r} f\right)_{1}(x)-c \leq 0$ for all $x \in \mathbb{R}_{+}$and, therefore, that the opportunity is worthless and never exercised. This completes our proof.

Part (A) of Proposition 1 states a set of conditions under which the relationship between post-exercise volatility and the value of the investment opportunity is unambiguously positive. It is worth emphasizing that in the present case this does not necessarily imply that increased post-exercise uncertainty would postpone rational exercise by expanding the continuation region, since increased post-exercise uncertainty increases the exercise payoff as well and, therefore, the relationship between post-exercise volatility and the optimal policy is a priori ambiguous. Part (B) of Proposition 1 then, in turn, states a set of conditions under which the sign of the relationship between post-exercise volatility and the value of the investment opportunity is negative. As in the case of part (A) this finding does not, however, necessarily imply that increased post-exercise uncertainty should speed up rational exercise since increased post-exercise uncertainty decreases the exercise payoff as well. Finally, part (C) of Proposition 1 states the intuitively clear result that an investment opportunity will never be exercised if the post-exercise revenue flow is negative. In other words, an investment opportunity may be exercised only if it results in an improved expected cash flow. Otherwise, adoption is clearly suboptimal and the investment opportunity is left unutilized.

Before proceeding any further in the analysis of the stopping problem (1), we first consider the associated optimal stopping problem

$$
H_{i}(x)=\sup _{\tau} \mathbb{E}_{x}\left[e^{-r \tau}\left(\left(R_{r} g\right)_{i}\left(X_{i}(\tau)\right)-c\right)\right],
$$

where $\tau$ is an arbitrary stopping time, and $g \in \mathcal{L}_{i}^{1}(\mathbb{P}) \cap C\left(\mathbb{R}_{+}\right)$. It is well-known from the classical theory of diffusions that if $g \in \mathcal{L}_{i}^{1}(\mathbb{P}) \cap C\left(\mathbb{R}_{+}\right)$, then (cf. Mandl (1968))

$$
\begin{aligned}
\left(R_{r} g\right)_{i}(x) & =\frac{2}{\sigma_{i}^{2}\left(\psi_{i}-\varphi_{i}\right)}\left[x^{\varphi_{i}} \int_{0}^{x} y^{-1-\varphi_{i}} g(y) d y+x^{\psi_{i}} \int_{x}^{\infty} y^{-1-\psi_{i}} g(y) d y\right] \\
& =\frac{2}{\sigma_{i}^{2}\left(\psi_{i}-\varphi_{i}\right)}\left[\int_{0}^{1} u^{-1-\varphi_{i}} g(u x) d u+\int_{1}^{\infty} u^{-1-\psi_{i}} g(u x) d u\right]
\end{aligned}
$$

where

$$
\psi_{i}=\frac{1}{2}-\frac{\mu}{\sigma_{i}^{2}}+\sqrt{\left(\frac{1}{2}-\frac{\mu}{\sigma_{i}^{2}}\right)^{2}+\frac{2 r}{\sigma_{i}^{2}}}>0
$$


denotes the positive and

$$
\varphi_{i}=\frac{1}{2}-\frac{\mu}{\sigma_{i}^{2}}-\sqrt{\left(\frac{1}{2}-\frac{\mu}{\sigma_{i}^{2}}\right)^{2}+\frac{2 r}{\sigma_{i}^{2}}}<0
$$

denotes the negative root of the characteristic equation $\sigma_{i}^{2} a(a-1) / 2+\mu a-r=0$ of Euler's differential equation $\left(\left(\mathcal{A}_{i}-r\right) u\right)(x)=0$. Especially,

$$
\frac{d}{d x}\left[x^{-\varphi_{i}}\left(\left(R_{r} g\right)_{i}(x)-c\right)\right]=\frac{2}{\sigma_{i}^{2}} x^{\psi_{i}-\varphi_{i}-1} \int_{x}^{\infty} y^{-1-\psi_{i}}(g(y)-r c) d y
$$

and

$$
\frac{d}{d x}\left[x^{-\psi_{i}}\left(\left(R_{r} g\right)_{i}(x)-c\right)\right]=-\frac{2}{\sigma_{i}^{2}} x^{\varphi_{i}-\psi_{i}-1} \int_{0}^{x} y^{-1-\varphi_{i}}(g(y)-r c) d y .
$$

We can now demonstrate that

Lemma 3. (A) Assume that $g \in \mathcal{L}_{i}^{1}(\mathbb{P}) \cap C\left(\mathbb{R}_{+}\right)$is non-decreasing and satisfies the conditions $\lim _{x \downarrow 0} g(x)<r c$ and $\lim _{x \rightarrow \infty} g(x)>r c$. Then,

$$
H_{i}(x)=x^{\psi_{i}} \sup _{y \geq x}\left\{y^{-\psi_{i}}\left(\left(R_{r} g\right)_{i}(y)-c\right)\right\}= \begin{cases}\left(R_{r} g\right)_{i}(x)-c, & x \geq x_{i}^{*} \\ \left(\left(R_{r} g\right)_{i}\left(x_{i}^{*}\right)-c\right)\left(x / x_{i}^{*}\right)^{\psi_{i}}, & x<x_{i}^{*},\end{cases}
$$

and the optimal stopping time is $\tau\left(x_{i}^{*}\right)=\inf \left\{t \geq 0: X_{i}(t) \geq x_{i}^{*}\right\}$, where the optimal stopping boundary $x_{i}^{*}=\operatorname{argmax}\left\{x^{-\psi_{i}}\left(\left(R_{r} g\right)_{i}(x)-c\right)\right\} \in\left(g^{-1}(r c), \infty\right)$ is the unique root of the equation

$$
\int_{0}^{x_{i}^{*}} y^{-1-\varphi_{i}}(g(y)-r c) d y=0 .
$$

(B) Assume that $g \in \mathcal{L}_{i}^{1}(\mathbb{P}) \cap C\left(\mathbb{R}_{+}\right)$is non-increasing and satisfies the conditions $\lim _{x \downarrow 0} g(x)>$ rc and $\lim _{x \rightarrow \infty} g(x)<$ rc. Then,

$$
H_{i}(x)=x^{\varphi_{i}} \sup _{y \leq x}\left\{y^{-\varphi_{i}}\left(\left(R_{r} g\right)_{i}(y)-c\right)\right\}= \begin{cases}\left(\left(R_{r} g\right)_{i}\left(\tilde{x}_{i}\right)-c\right)\left(x / \tilde{x}_{i}\right)^{\varphi_{i}}, & x>\tilde{x}_{i}, \\ \left(R_{r} g\right)_{i}(x)-c, & x \leq \tilde{x}_{i}\end{cases}
$$

and the optimal stopping time is $\tau\left(\tilde{x}_{i}\right)=\inf \left\{t \geq 0: X_{i}(t) \leq \tilde{x}_{i}\right\}$, where the optimal stopping boundary $\tilde{x}_{i}=\operatorname{argmax}\left\{x^{-\varphi_{i}}\left(\left(R_{r} g\right)_{i}(x)-c\right)\right\} \in\left(0, g^{-1}(r c)\right)$ is the unique root of the equation

$$
\int_{\tilde{x}_{i}}^{\infty} y^{-1-\psi_{i}}(g(y)-r c) d y=0 .
$$

Proof. (A) Denote the proposed value function (13) as $\hat{H}_{i}(x)$. Since

$$
\hat{H}_{i}(x)=\mathbb{E}_{x}\left[e^{-r \tau\left(x_{i}^{*}\right)}\left(\left(R_{r} g\right)_{i}\left(X_{i}\left(\tau\left(x_{i}^{*}\right)\right)\right)-c\right)\right],
$$

and the stopping time in (9) is arbitrary, we find that $H_{i}(x) \geq \hat{H}_{i}(x)$ for all $x \in \mathbb{R}_{+}$. To prove the opposite inequality, we first observe that the proposed value function $\hat{H}_{i}(x)$ is continuous on $\mathbb{R}_{+}$. We now have to prove that (14) has a unique root on $\mathbb{R}_{+}$. To accomplish this task, we first define the functional $I_{1}: \mathbb{R}_{+} \mapsto \mathbb{R}$ as

$$
I_{1}(x)=\int_{0}^{x} y^{-1-\varphi_{i}}(g(y)-r c) d y
$$


and observe that $I(x)<0$ as long as $g(x) \leq r c$, and that $I_{1}^{\prime}(x)=x^{-1-\varphi_{i}}(g(x)-r c)>0$ when $g(x)>r c$. If $K>g^{-1}(r c)$, then the monotonicity of $g(x)$ implies that

$$
I_{1}(x)=I_{1}(K)+\int_{K}^{x} y^{-1-\varphi_{i}}(g(y)-r c) d y \geq I_{1}(K)-\frac{(g(K)-r c)}{\varphi_{i}}\left[x^{-\varphi_{i}}-K^{-\varphi_{i}}\right] \rightarrow \infty,
$$

as $x \rightarrow \infty$. Combining this finding with the continuity and monotonicity properties of the functional $I_{1}(x)$ on $\mathbb{R}_{+}$then proves that $I_{1}(x)=0$ has a unique root $x_{i}^{*} \in\left(g^{-1}(r c), \infty\right)$. Equation (12) then in turn implies that $x_{i}^{*}=\operatorname{argmax}\left\{x^{-\psi_{i}}\left(\left(R_{r} g\right)_{i}(x)-c\right)\right\}$. Given these findings, we observe that the proposed value function $\hat{H}_{i}(x)$ dominates the exercise payoff $\left(R_{r} g\right)_{i}(x)-c$ and is non-negative, since

$$
x_{i}^{*-\psi_{i}}\left(\left(R_{r} g\right)_{i}\left(x_{i}^{*}\right)-c\right)=\frac{2}{\sigma_{i}^{2}\left(\psi_{i}-\varphi_{i}\right)} \int_{x_{i}^{*}}^{\infty} y^{-1-\psi_{i}}(g(y)-r c) d y>0 .
$$

Moreover, since $\hat{H}_{i}(x)$ is non-decreasing, $x_{i}^{*}=\operatorname{argmax}\left\{x^{-\psi_{i}}\left(\left(R_{r} g\right)_{i}(x)-c\right)\right\}$, and $\hat{H}_{i}(x)$ can be rewritten as

$$
\hat{H}_{i}(x)=x^{\psi_{i}} \sup _{y \geq x}\left\{y^{-\psi_{i}}\left(\left(R_{r} g\right)_{i}(y)-c\right)\right\}
$$

we find that

$$
\frac{\hat{H}_{i}(x)}{\hat{H}_{i}(y)} \geq \min \left((x / y)^{\psi_{i}},(x / y)^{\varphi_{i}}\right)
$$

for all $x, y \in \mathbb{R}_{+}$. Thus, the proposed value function constitutes a $r$-excessive majorant of the payoff $\left(R_{r} g\right)_{i}(x)-c$ (cf. Borodin and Salminen (1996)) for the geometric Brownian motion $X_{i}(t)$. Since $H_{i}(x)$ is the least of such majorants, we find that $H_{i}(x) \leq \hat{H}_{i}(x)$, thus completing the proof of part (A). The proof of part (B) is completely analogous.

In order to apply the results of Lemma 3, we have to rewrite the objective functional in (8) in another form. We observe from the proof of Lemma 2 that for all $x \in \mathbb{R}_{+}$we have by invoking the strong Markov property of diffusions that

$$
\begin{aligned}
\left.\mathbb{E}_{x}\left[e^{-r T^{*}}\left(\left(R_{r} f\right)_{2}-\left(R_{r} f\right)_{1}\right)\left(X_{1}\left(T^{*}\right)\right)\right)\right] & =\left(R_{r} f\right)_{2}(x)-\left(R_{r} f\right)_{1}(x) \\
& +\mathbb{E}_{x} \int_{0}^{T^{*}} e^{-r s} \frac{1}{2}\left(\sigma_{1}^{2}-\sigma_{2}^{2}\right) X_{1}^{2}(s)\left(R_{r} f\right)_{2}^{\prime \prime}\left(X_{1}(s)\right) d s,
\end{aligned}
$$

where $T^{*}=\min \left(T_{n}, \tau\right)$ and $\tau$ is a stopping time. Letting $n \rightarrow \infty$ then shows that (8) can be rewritten as

$$
V(x)=\left(R_{r} f\right)_{2}(x)-c+\sup _{\tau} \mathbb{E}_{x} \int_{0}^{\tau} e^{-r s} h\left(X_{1}(s)\right) d s,
$$

where $h(x)=r c-\frac{1}{2}\left(\sigma_{2}^{2}-\sigma_{1}^{2}\right) x^{2}\left(R_{r} f\right)_{2}^{\prime \prime}(x)$. Let us now consider the mapping $x^{2}\left(R_{r} f\right)_{2}^{\prime \prime}(x)$ more closely. It is clear from (10) that

$\frac{1}{2} \sigma_{2}^{2} x^{2}\left(R_{r} f\right)_{2}^{\prime \prime}(x)=\frac{\varphi_{2}\left(\varphi_{2}-1\right)}{\left(\psi_{2}-\varphi_{2}\right)} \int_{0}^{1} u^{-1-\varphi_{2}} f(u x) d u+\frac{\psi_{2}\left(\psi_{2}-1\right)}{\left(\psi_{2}-\varphi_{2}\right)} \int_{1}^{\infty} u^{-1-\psi_{2}} f(u x) d u-f(x)$.

A straightforward consequence of this result is that whenever $\lim _{x \downarrow} f(x)=f(0)<\infty$ we have that $\lim _{x \downarrow 0} x^{2}\left(R_{r} f\right)_{2}^{\prime \prime}(x)=0$.

Our main result summarizing the optimal risk adoption policy is now summarized in the following proposition. 
Proposition 2. Assume that $f(0)<\infty, f \in C^{2}\left(\mathbb{R}_{+}\right),\left(\sigma_{2}^{2}-\sigma_{1}^{2}\right) f^{\prime \prime}(x) \geq 0, \frac{1}{2}\left(\sigma_{2}^{2}-\sigma_{1}^{2}\right) x^{2} f^{\prime \prime}(x)$ is non-decreasing, and $\lim _{x \rightarrow \infty} \frac{1}{2}\left(\sigma_{2}^{2}-\sigma_{1}^{2}\right) x^{2} f^{\prime \prime}(x)=\infty$. Then, the value of the optimal risk adoption policy is

$$
V(x)= \begin{cases}\left(R_{r} f\right)_{2}(x)-c, & x \geq x^{*} \\ \left(R_{r} f\right)_{1}(x)+\left(\left(R_{r} f\right)_{2}\left(x^{*}\right)-\left(R_{r} f\right)_{1}\left(x^{*}\right)-c\right)\left(x / x^{*}\right)^{\psi_{1}}, & x<x^{*}\end{cases}
$$

where $x^{*} \in h^{-1}\left(\mathbb{R}_{-}\right)$is the unique root of the equation

$$
\int_{0}^{x^{*}} y^{-1-\varphi_{1}}\left(r c-\frac{1}{2}\left(\sigma_{2}^{2}-\sigma_{1}^{2}\right) y^{2}\left(R_{r} f\right)_{2}^{\prime \prime}(y)\right) d y=0,
$$

and $\tau\left(x^{*}\right)=\inf \left\{t \geq 0: X(t) \geq x^{*}\right\}$ is the optimal stopping date.

Proof. Define the functional $u_{i}: \mathbb{R}_{+}^{2} \mapsto \mathbb{R}$ as $u_{i}(t, x)=\mathbb{E}\left[f\left(X_{i}^{x}(t)\right)\right]=\mathbb{E}\left[f\left(x e^{\mu t} Z_{i}(t)\right)\right]$, where $Z_{i}(t)=e^{\sigma_{i} W(t)-\frac{1}{2} \sigma_{i}^{2} t}$ is a positive $\mathcal{F}_{t}$-martingale. If $f \in C^{2}\left(\mathbb{R}_{+}\right)$, then

$$
x^{2} \frac{\partial^{2} u_{i}}{\partial x^{2}}(t, x)=x^{2} \mathbb{E}\left[e^{2 \mu t} Z_{i}^{2}(t) f^{\prime \prime}\left(x e^{\mu t} Z_{i}(t)\right)\right]=\mathbb{E}\left[\left(X_{i}^{x}(t)\right)^{2} f^{\prime \prime}\left(X_{i}^{x}(t)\right)\right]
$$

implying that

$$
\frac{1}{2}\left(\sigma_{2}^{2}-\sigma_{1}^{2}\right) x^{2} \frac{\partial^{2} u_{i}}{\partial x^{2}}(t, x)=\frac{1}{2} \mathbb{E}\left[\left(\sigma_{2}^{2}-\sigma_{1}^{2}\right) X_{i}^{x 2}(t) f^{\prime \prime}\left(X_{i}^{x}(t)\right)\right] \geq 0
$$

and that $h(x)$ is non-decreasing, since expectation preserves monotonicity. The assumption $f(0)<\infty$ then implies that $h(0)=r c$ and the assumption $\lim _{x \rightarrow \infty} \frac{1}{2}\left(\sigma_{2}^{2}-\sigma_{1}^{2}\right) x^{2} f^{\prime \prime}(x)=\infty$ guarantees that $h(x) \downarrow-\infty$ as $x \rightarrow \infty$. Thus, $h(x)$ has a unique root on $\mathbb{R}_{+}$. Invoking the strong Markov property of diffusions then yields that (17) can be rewritten as

$$
V(x)=\left(R_{r} f\right)_{2}(x)-c+\left(R_{r} h\right)_{1}(x)+\sup _{\tau} \mathbb{E}_{x}\left[e^{-r \tau}\left(R_{r} \tilde{h}\right)_{1}\left(X_{1}(\tau)\right)\right]
$$

where $\tilde{h}(x)=-h(x)$. The alleged results then follow from Lemma 3 and (8).

It is worth pointing out that (19) is the well-known smooth-fit condition requiring the differentiability of the value across the exercise boundary. In the present case, it can be alternatively written as

$$
\left(\left(R_{r} f\right)_{2}^{\prime}\left(x^{*}\right)-\left(R_{r} f\right)_{1}^{\prime}\left(x^{*}\right)\right) x^{*}=\psi_{1}\left(\left(R_{r} f\right)_{2}\left(x^{*}\right)-\left(R_{r} f\right)_{1}\left(x^{*}\right)-c\right) .
$$

Proposition 2 states a set of conditions under which an optimal risk adoption policy always exists. As is clear from our analysis, the monotonicity of the mapping $h(x)$ is the essential factor determining the existence and uniqueness of an optimal strategy. More precisely, as is clear from Lemma 3, an optimal exercise threshold $x^{*}$ always exists whenever $h(x)$ is non-decreasing and satisfies the conditions $\lim _{x \downarrow 0} h(x)<0$ and $\lim _{x \rightarrow \infty} h(x)>0$. Moreover, the smooth-fit condition (20) offers a characterization of the critical adoption cost, $c$, above which the decision maker is willing to shift the risk of the process from $\sigma_{1}$ to $\sigma_{2}$. At this critical adoption cost the firm is indifferent between switching to the new technology with risk characteristics $\sigma_{2}$ and holding on to the incumbent technology with risk characteristics $\sigma_{1}$.

From Proposition 2 we can conclude that the optimal risk adoption policy reflects an interplay between three factors: the incorporated risks as characterized by the relative magnitude of the risk measures $\left(\sigma_{1}\right.$ and $\left.\sigma_{2}\right)$, the adoption cost $(c)$ and the functional relationship between the state variable and the profit stream $(f)$. Intuitively, the optimal adoption timing, and thereby the optimal risk taking emerges as the interplay between two effects: 1 . 
the real option effect (i.e. the ability to postpone the exercise of an investment opportunity) and 2. the Jensen's inequality effect. As our results indicate, from the point of view of a decision maker endowed with a convex (concave) objective function increased post-adoption volatility tends to increase (decrease) the expected cumulative present value of the postadoption cash flow and, therefore, tends to decrease (increase) the option value of waiting, which consequently accelerates (decelerates) current investment.

Our result has strong implications for the relationship between delegation and risk taking. Through the design of incentive contracts, whereby the firm's decision making is delegated to an agent with an objective function different from the of the principal, the principal can implement shifts in the risk adoption policy of firms. For example, by implementing option contracts the decision making is delegated to an agent with a more convex objective function and in light of Proposition 2 the use of such a contract will generate increased risk taking in the sense of lowering the threshold for switching to a more risky technology. In this respect our prediction would be that an increased use of option contracts would imply increased corporate risk taking - a prediction which could, in principle, be empirically tested.

Illustration In order to illustrate explicitly the results of this section, assume that the mapping belongs into the $H A R A$-class, i.e. that $f(x)=\frac{1}{\gamma} x^{\gamma}, \gamma>0$. In that case,

$$
\left(R_{r} f\right)_{i}(x)=\frac{x^{\gamma}}{\gamma\left(r-\rho\left(\sigma_{i}\right)\right)}
$$

provided that $r>\rho\left(\sigma_{i}\right)$, where $\rho\left(\sigma_{i}\right)=\gamma \mu+\frac{1}{2} \sigma_{i}^{2} \gamma(\gamma-1), i=1,2$. Thus, if $r>$ $\max \left(\rho\left(\sigma_{1}\right), \rho\left(\sigma_{2}\right)\right)$ we find that (8) can be written as

$$
V(x)=\frac{x^{\gamma}}{\gamma\left(r-\rho\left(\sigma_{1}\right)\right)}+\sup _{\tau} \mathbb{E}_{x}\left[e^{-r \tau}\left(\frac{(\gamma-1)\left(\sigma_{2}^{2}-\sigma_{1}^{2}\right)}{2\left(r-\rho\left(\sigma_{2}\right)\right)\left(r-\rho\left(\sigma_{1}\right)\right)} X_{1}^{\gamma}(\tau)-c\right)\right] .
$$

Moreover, in the present application it holds that

$$
h(x)=r c-\frac{1}{2}\left(\sigma_{2}^{2}-\sigma_{1}^{2}\right) \frac{(\gamma-1) x^{\gamma}}{\left(r-\rho\left(\sigma_{2}\right)\right)} .
$$

Invoking (19) as well as the inequality $(\gamma-1)\left(\sigma_{2}-\sigma_{1}\right) \geq 0$, and applying the relations $\sigma_{i}^{2}\left(\psi_{i}-\gamma\right)\left(\gamma-\varphi_{i}\right)=2\left(r-\rho\left(\sigma_{i}\right)\right)$ and $-2 r=\sigma_{i}^{2} \psi_{i} \varphi_{i}$ then yield that the optimal exercise threshold is

$$
x^{*}\left(\sigma_{1}, \sigma_{2}\right)=\left[\frac{\psi_{1} c}{\psi_{1}-\gamma} \frac{2\left(r-\rho\left(\sigma_{1}\right)\right)\left(r-\rho\left(\sigma_{2}\right)\right)}{(\gamma-1)\left(\sigma_{2}^{2}-\sigma_{1}^{2}\right)}\right]^{1 / \gamma},
$$

and the value of the optimal adoption policy reads as

$$
V(x)= \begin{cases}\frac{x^{\gamma}}{\gamma\left(r-\rho\left(\sigma_{1}\right)\right)}+\left(\frac{(\gamma-1)\left(\sigma_{2}^{2}-\sigma_{1}^{2}\right)}{2\left(r-\rho\left(\sigma_{2}\right)\left(r-\rho\left(\sigma_{1}\right)\right)\right.} x^{\gamma}-c\right), & x \geq x^{*}\left(\sigma_{1}, \sigma_{2}\right) \\ \frac{x^{\gamma}}{\gamma\left(r-\rho\left(\sigma_{1}\right)\right)}+\frac{\gamma c}{\psi_{1}-\gamma}\left(x / x^{*}\left(\sigma_{1}, \sigma_{2}\right)\right)^{\psi_{1}}, & x<x^{*}\left(\sigma_{1}, \sigma_{2}\right) .\end{cases}
$$

Two interesting implications of the definition of the optimal threshold are that

$$
\frac{\partial x^{*}\left(\sigma_{1}, \sigma_{2}\right)}{\partial \sigma_{2}}=\frac{1}{1-\gamma}\left[\frac{4 \psi_{1} \sigma_{2}\left(r-\rho\left(\sigma_{1}\right)\right)^{2}}{\left(\psi_{1}-\gamma\right)\left(\sigma_{2}^{2}-\sigma_{1}^{2}\right)^{2}}\right] \stackrel{<}{<} 0, \text { if } 1 \underset{<}{<} \gamma
$$

and

$$
\frac{\partial x^{*}\left(\sigma_{1}, \sigma_{2}\right)}{\partial \sigma_{1}}=\left[\frac{\left(r-\rho\left(\sigma_{2}\right)\right) 2 r}{(\gamma-1)\left(\sigma_{2}^{2}-\sigma_{1}^{2}\right)}\right]\left[\frac{2 \gamma\left(\varphi_{1}-1\right)}{\varphi_{1} \sigma_{1}\left(\psi_{1}-\varphi_{1}\right)}+\left(1-\frac{\gamma}{\varphi_{1}}\right) \frac{2 \sigma_{1}}{\left(\sigma_{2}^{2}-\sigma_{1}^{2}\right)}\right] .
$$


We observe from (21) that increased post-exercise volatility $\sigma_{2}$ increases the optimal exercise threshold $x^{*}\left(\sigma_{1}, \sigma_{2}\right)$ only when the mapping $f(x)$ is strictly concave, that is, when $\gamma \in(0,1)$. If this is indeed the case, then

$$
\lim _{\sigma_{2} \downarrow 0} x^{*}\left(\sigma_{1}, \sigma_{2}\right)=\left[\frac{\psi_{1} c}{\psi_{1}-\gamma} \frac{2\left(r-\rho\left(\sigma_{1}\right)\right)(r-\mu \gamma)}{(1-\gamma) \sigma_{1}^{2}}\right]^{1 / \gamma}
$$

and

$$
\lim _{\sigma_{2} \uparrow \sigma_{1}} x^{*}\left(\sigma_{1}, \sigma_{2}\right)=\infty .
$$

Consequently, we observe that when $f(x)$ is concave the option is not exercised instantaneously even when the post-exercise situation is deterministic (i.e. $\sigma_{2}=0$ ). Interestingly, we observe that whenever $f(x)$ is convex (a property which is typically satisfied by profit functions), increased post-exercise volatility decreases the optimal exercise threshold and, therefore, accelerates rational investment.

As is clear from (22), the impact of increased pre-exercise volatility $\sigma_{1}$ on the optimal threshold $x^{*}\left(\sigma_{1}, \sigma_{2}\right)$ is rather involved. Somewhat surprisingly, its sign does not always depend on the parameter $\gamma$ measuring the convexity or concavity of the mapping $f(x)$. Since the multiplier of the right hand side of $(22)$ and the first term on the brackets is positive, we notice that increased pre-exercise volatility increases the optimal threshold $x^{*}\left(\sigma_{1}, \sigma_{2}\right)$ whenever $\sigma_{2}>\sigma_{1}$. Since in that case adoption occurs only if $f(x)$ is convex, i.e., when $\gamma>1$, it follows that increased pre-exercise volatility increases the optimal threshold at which adoption is optimal whenever $\gamma>1$. However, if $\sigma_{2}<\sigma_{1}$ and $\gamma<1$, then the sign of (22) cannot be determined easily. However, numerical experimentation indicates that it should be negative. That is, increased pre-exercise uncertainty appears to accelerate investment whenever the mapping $f(x)$ is concave. Moreover, we find that if $\sigma_{2}<\sigma_{1}$ and $\gamma<1$, then

$$
\lim _{\sigma_{1} \downarrow \sigma_{2}} x^{*}\left(\sigma_{1}, \sigma_{2}\right)=\infty
$$

and

$$
\lim _{\sigma_{1} \uparrow \infty} x^{*}\left(\sigma_{1}, \sigma_{2}\right)=\left(\frac{\gamma c\left(r-\rho_{2}(\gamma)\right)}{(1-\gamma)}\right)^{1 / \gamma}
$$

We illustrate the optimal threshold in Figure 1 under the assumption that $\gamma=0.5, r=5 \%$, $\mu=4 \%, \sigma_{2}=0.1$, and $c=1$.

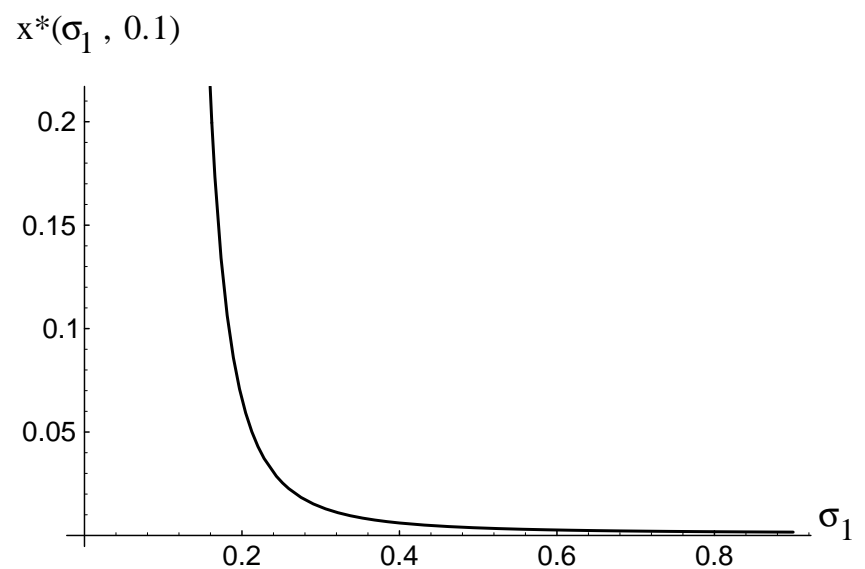

Figure 1: The Optimal Investment Threshold $x^{*}\left(\sigma_{1}, 0.1\right)$ 
These results are of essential interest, since they demonstrate that the standard argument appearing in the literature on real options, stating that increased volatility should decelerate investment, is essentially caused by the assumed stationarity of the underlying volatility structure, that is, by the assumption that the fluctuations of the underlying state variable are not affected by the investment decision itself. As our model clearly indicates, the impact of increased uncertainty on rational investment needs not be decelerating, provided that the underlying volatility is made endogenous (in the sense that the timing of the switch from one risk regime to another is optimized). This mechanism is probably one of the most important factors explaining the difficulties experienced in empirical studies when assessing whether the relationship between uncertainty and investment is positive or negative. Models neglecting this feature, whereby the exercise of an investment option endogenously creates information, overlook a key factor. The absence of this mechanism seems to be a crucial source for the common results with an inverse relationship between investment and uncertainty.

\section{Generalized Stochastic Dynamics}

It is our purpose in this section to extend the results of the previous section to the case where the underlying coupled process is more general than just the ordinary geometric Brownian motion. That is, in this section we plan to consider the valuation problem (8) under a more general coupled diffusion describing the underlying stochastic dynamics. In order to accomplish this task, we now assume that the underlying process $\{X(t) ; t \geq 0\}$ evolves according to the dynamics (4), where

$$
d X_{i}(t)=\mu\left(X_{i}(t)\right) d t+\sigma_{i}\left(X_{i}(t)\right) d W(t),
$$

$X_{1}(0)=x$, and $X_{1}(\tau-)=X_{2}(\tau+)$. We assume that the infinitesimal coefficients $\mu: \mathbb{R}_{+} \mapsto$ $\mathbb{R}, \sigma_{1}: \mathbb{R}_{+} \mapsto \mathbb{R}$, and $\sigma_{2}: \mathbb{R}_{+} \mapsto \mathbb{R}$ are continuously differentiable with Lipschitz-continuous derivatives on $\mathbb{R}_{+}$, and that 0 and $\infty$ are natural for the diffusions $X_{1}(t)$ and $X_{2}(t)$. Thus, we assume that although the underlying process $X$ may tend towards a boundary, it will never attain it in finite expected time. In line with the notation applied in the previous section, we denote as $\mathcal{L}_{i}^{1}(\mathbb{P})$ the class of measurable mappings satisfying the absolute integrability condition (7) (given the dynamics in (23)) and denote as $\hat{\mathcal{A}}_{i}$ the differential operator

$$
\hat{\mathcal{A}}_{i}=\frac{1}{2} \sigma_{i}^{2}(x) \frac{d^{2}}{d x^{2}}+\mu(x) \frac{d}{d x}
$$

representing the infinitesimal generator of the diffusion $X_{i}(t), i=1,2$. Given our technical assumptions, we are now in position to prove the following.

Lemma 4. (A) Assume that for all $(t, x) \in \mathbb{R}_{+}^{2}$

$$
\mathbb{E}_{x}\left[\exp \left(\int_{0}^{t}{\sigma^{\prime}}_{i}^{2}\left(X_{i}(t)\right) d t\right)\right]<\infty,
$$

that $\mu(x)$ is convex, and that $f \in \mathcal{L}_{i}^{1}(\mathbb{P})$ is convex and non-decreasing. Then, $\left(R_{r} f\right)_{i}(x)$ is non-decreasing and convex on $\mathbb{R}_{+}$. Moreover, increased volatility increases the expected cumulative present value of the revenue flow $f(x)$. That is, if $f \in \mathcal{L}_{1}^{1}(\mathbb{P}) \cap \mathcal{L}_{2}^{1}(\mathbb{P})$ and $\sigma_{2}(x) \geq \sigma_{1}(x)$, then $\left(R_{r} f\right)_{2}(x) \geq\left(R_{r} f\right)_{1}(x)$ for all $x \in \mathbb{R}_{+}$.

(B) Assume that (25) is satisfied, that $\mu(x)$ is concave and that $f \in \mathcal{L}_{i}^{1}(\mathbb{P})$ is concave and non-decreasing. Then, $\left(R_{r} f\right)_{i}(x)$ is non-decreasing and concave on $\mathbb{R}_{+}$. Moreover, increased volatility decreases the expected cumulative present value of the revenue flow $f(x)$. That is, if $f \in \mathcal{L}_{1}^{1}(\mathbb{P}) \cap \mathcal{L}_{2}^{1}(\mathbb{P})$ and $\sigma_{2}(x) \geq \sigma_{1}(x)$, then $\left(R_{r} f\right)_{2}(x) \leq\left(R_{r} f\right)_{1}(x)$ for all $x \in \mathbb{R}_{+}$.

(C) Assume that (25) is satisfied, that $\mu(x)$ is concave and that $f \in \mathcal{L}_{i}^{1}(\mathbb{P})$ is convex and 
non-increasing. Then, $\left(R_{r} f\right)_{i}(x)$ is non-increasing and convex on $\mathbb{R}_{+}$. Moreover, increased volatility increases the expected cumulative present value of the revenue flow $f(x)$. That is, if $f \in \mathcal{L}_{1}^{1}(\mathbb{P}) \cap \mathcal{L}_{2}^{1}(\mathbb{P})$ and $\sigma_{2}(x) \geq \sigma_{1}(x)$, then $\left(R_{r} f\right)_{2}(x) \geq\left(R_{r} f\right)_{1}(x)$ for all $x \in \mathbb{R}_{+}$.

(D) Assume that (25) is satisfied, that $\mu(x)$ is convex and that $f \in \mathcal{L}_{i}^{1}(\mathbb{P})$ is concave and non-increasing. Then, $\left(R_{r} f\right)_{i}(x)$ is non-increasing and concave on $\mathbb{R}_{+}$. Moreover, increased volatility decreases the expected cumulative present value of the revenue flow $f(x)$. That is, if $f \in \mathcal{L}_{1}^{1}(\mathbb{P}) \cap \mathcal{L}_{2}^{1}(\mathbb{P})$ and $\sigma_{2}(x) \geq \sigma_{1}(x)$, then $\left(R_{r} f\right)_{2}(x) \leq\left(R_{r} f\right)_{1}(x)$ for all $x \in \mathbb{R}_{+}$.

Proof. (A) The convexity of the mapping $f(x)$ implies that there is a sequence of continuously differentiable convex mappings $f_{n}(x)$ converging (from below) uniformly on compacts to $f(x)$ $\left(f_{n}(x)\right.$ is a mollification of $f(x)$, cf. Alvarez (2001) and Protter (1990), p. 163). Consider now the functional

$$
\left(R_{r} f_{n}\right)_{i}(x)=\mathbb{E}_{x} \int_{0}^{\infty} e^{-r s} f_{n}\left(X_{i}^{x}(s)\right) d s .
$$

It is then clear that (cf. Protter (1990), pp. 250-251)

$$
\left(R_{r} f_{n}\right)_{i}^{\prime}(x)=\mathbb{E}_{x} \int_{0}^{\infty} e^{-\int_{0}^{t}\left(r-\mu^{\prime}\left(X_{i}^{x}(s)\right)\right) d s} f_{n}^{\prime}\left(X_{i}^{x}(t)\right) M(t) d t
$$

where

$$
M(t)=\exp \left(\int_{0}^{t} \sigma^{\prime}\left(X_{i}^{x}(s)\right) d W(s)-\frac{1}{2} \int_{0}^{t} \sigma^{\prime 2}\left(X_{i}^{x}(s)\right) d s\right)
$$

is a positive martingale given the Novikov-condition (25). The convexity of $\left(R_{r} f_{n}\right)_{i}(x)$ follows then from Theorem 3 in Alvarez and Stenbacka (2001). Assume now that $\lambda \in[0,1]$ and that $x, y \in \mathbb{R}_{+}$. Then

$$
\left(R_{r} f\right)_{i}(\lambda x+(1-\lambda) y) \geq\left(R_{r} f_{n}\right)_{i}(\lambda x+(1-\lambda) y) \geq \lambda\left(R_{r} f_{n}\right)_{i}(x)+(1-\lambda)\left(R_{r} f_{n}\right)_{i}(y) .
$$

Letting now $n \rightarrow \infty$ and invoking monotonic convergence then yields that

$$
\left(R_{r} f\right)_{i}(\lambda x+(1-\lambda) y) \geq \lambda\left(R_{r} f\right)_{i}(x)+(1-\lambda)\left(R_{r} f\right)_{i}(y),
$$

for all $x, y \in \mathbb{R}_{+}$and $\lambda \in[0,1]$. The rest of the proof of part (A) of our lemma follows directly from Theorem 3 in Alvarez and Stenbacka (2001). The proof of parts (B), (C), and (D) are completely analogous.

Lemma 4 states a set of conditions under which the convexity or the concavity of the expected cumulative present value of the revenue flow $f(x)$ can always be unambiguously established. Consequently, Lemma 4 states a set of conditions under which the sign of the relationship between increased volatility and the expected cumulative present value of the revenue flow $f(x)$ can be determined. It is worth observing that our results clearly indicate that contrary to the case where the underlying diffusion evolves according to a standard geometric Brownian motion the convexity (or concavity) of the expected cumulative present value $\left(R_{r} f\right)(x)$ does not only depend on the convexity (or concavity) of the revenue flow $f(x)$, it is also affected by the form of the infinitesimal drift $\mu(x)$. An important consequence of Lemma 4 extending the results of our Proposition 1 is now summarized in the following.

Proposition 3. (A) Assume that $f \in \mathcal{L}_{1}^{1}(\mathbb{P}) \cap \mathcal{L}_{2}^{1}(\mathbb{P})$, that $\left(R_{r} f\right)_{i}(x) i=1,2$ is convex, and that $\sigma_{1}(x)<\sigma_{2}(x)$. Then, increased post-exercise volatility $\sigma_{2}(x)$ increases the value $V(x)$. (B) Assume that $f \in \mathcal{L}_{1}^{1}(\mathbb{P}) \cap \mathcal{L}_{2}^{1}(\mathbb{P})$, that $\left(R_{r} f\right)_{i}(x) i=1,2$ is concave, and that $\sigma_{1}(x)>$ $\sigma_{2}(x)$. Then, increased post-exercise volatility $\sigma_{2}(x)$ decreases the value $V(x)$.

(C) Assume that $f \in \mathcal{L}_{1}^{1}(\mathbb{P})$ and that either $\left(R_{r} f\right)_{i}(x) i=1,2$ is convex and $\sigma_{2}(x) \leq \sigma_{1}(x)$, or that $\left(R_{r} f\right)_{i}(x) i=1,2$ is concave and $\sigma_{2}(x) \geq \sigma_{1}(x)$. Then, the option is never exercised (i.e. $\tau=\infty$ ) and $V(x)=\left(R_{r} f\right)_{1}(x)$ for all $x \in \mathbb{R}_{+}$. 
Proof. Analogous with the proof of Proposition 1.

Proposition 3 states a set of conditions under which the sign of the relationship between volatility and the value of the investment opportunity can be unambiguously determined. Given the results of our Lemma 4, we observe that if the conditions of either part (A) or part (C) of Lemma 4 are met and $\sigma_{1}(x)<\sigma_{2}(x)$, then increased post-exercise volatility $\sigma_{2}(x)$ increases the value $V(x)$. Similarly, we find that if the conditions of either part (B) or part (D) of Lemma 4 are met and $\sigma_{1}(x)>\sigma_{2}(x)$, then increased post-exercise volatility $\sigma_{2}(x)$ decreases the value $V(x)$.

Since the boundaries of the state-space of the diffusions $X_{i}$ were assumed to be natural, we find that the expected cumulative present value $\left(R_{r} f\right)_{i}(x)$ of the revenue flow $f(x)$ is the unique bounded solution of the ordinary second-order differential equation $\left(\left(\mathcal{A}_{i}-\right.\right.$ $\left.r)\left(R_{r} f\right)_{i}\right)(x)+f(x)=0$. Consequently, we find that $\left(\left(\mathcal{A}_{1}-r\right)\left(R_{r} f\right)_{2}\right)(x)-\left(\left(\mathcal{A}_{1}-r\right)\left(R_{r} f\right)_{1}\right)(x)=$ $\left(\left(\mathcal{A}_{1}-\mathcal{A}_{2}\right)\left(R_{r} f\right)_{2}\right)(x)$. Invoking now Dynkin's theorem yields that (8) can be rewritten as

$$
V(x)=\left(R_{r} f\right)_{2}(x)-c+\sup _{\tau} \mathbb{E}_{x} \int_{0}^{\tau} e^{-r s} H\left(X_{1}(s)\right) d s,
$$

where $H(x)=r c-\frac{1}{2}\left(\sigma_{2}^{2}(x)-\sigma_{1}^{2}(x)\right)\left(R_{r} f\right)_{2}^{\prime \prime}(x)$ can be interpreted as the instantaneous premium of waiting. Thus, we find that the considered optimal adoption problem can be expressed in a similar form as in the previous section. We can state the following.

Proposition 4. Assume that $\frac{1}{2}\left(\sigma_{2}^{2}(x)-\sigma_{1}^{2}(x)\right)\left(R_{r} f\right)_{2}^{\prime \prime}(x) \geq 0$ and that $H(x)$ is non-increasing and satisfies the conditions

$$
\lim _{x \downarrow 0} H(x)>0>\lim _{x \rightarrow \infty} H(x) .
$$

Then there is a unique optimal exercise threshold $x^{*}$ satisfying the optimality condition

$$
\int_{0}^{x^{*}} \psi_{1}(y) H(y) m_{1}^{\prime}(y) d y=0,
$$

where $\psi_{1}(x)$ denotes the increasing fundamental solution of the ordinary second-order differential equation $\left(\left(\mathcal{A}_{1}-r\right) u\right)(x)=0, m_{1}^{\prime}(x)=2 /\left(\sigma_{1}^{2}(x) S_{1}^{\prime}(x)\right)$ denotes the density of the speed measure of the diffusion $X_{1}$, and

$$
S_{1}^{\prime}(x)=\exp \left(\int \frac{2 \mu(x) d x}{\sigma_{1}^{2}(x)}\right)
$$

denotes the density of the scale function of the diffusion $X_{1}$. Moreover, the value of the investment opportunity reads as

$$
V(x)= \begin{cases}\left(\tilde{R}_{r} f\right)(x)-c, & x \geq x^{*} \\ \left(R_{r} f\right)(x)+\left(\left(\tilde{R}_{r} f\right)\left(x^{*}\right)-\left(R_{r} f\right)\left(x^{*}\right)-c\right) \frac{\psi(x)}{\psi\left(x^{*}\right)}, & x<x^{*} .\end{cases}
$$

Proof. The proof is analogous with the proof of Theorem 1 in Alvarez and Stenbacka (2001).

Unfortunately, in general it is very difficult, if possible at all, to characterize exactly the behavior of the mapping $\frac{1}{2}\left(\sigma_{2}^{2}(x)-\sigma_{1}^{2}(x)\right)\left(R_{r} f\right)_{2}^{\prime \prime}(x)$. To illustrate this argument more explicitly, assume that the post-adoption volatility is a constant multiple of the pre-adoption volatility, that is, assume that $\sigma_{2}(x)=\theta \sigma_{1}(x)$, where $\theta \geq 0$. In that case, the instantaneous premium of waiting reads as

$$
H(x)=r c-\frac{1}{2}\left(\theta^{2}-1\right) \sigma_{1}^{2}(x)\left(R_{r} f\right)_{2}^{\prime \prime}(x) .
$$


However, since the expected cumulative present value $\left(R_{r} f\right)_{2}(x)$ satisfies the ordinary second order differential equation $\left(\left(\mathcal{A}_{2}-r\right)\left(R_{r} f\right)_{2}\right)(x)+f(x)=0$ we find that $H(x)$ can be rewritten as

$$
H(x)=r c-\frac{\left(\theta^{2}-1\right)}{\theta^{2}} \frac{1}{2}\left[r\left(R_{r} f\right)_{2}(x)-\mu(x)\left(R_{r} f\right)_{2}^{\prime}(x)-f(x)\right] .
$$

Consequently, we observe that

$$
H^{\prime}(x)=-\frac{\left(\theta^{2}-1\right)}{\theta^{2}} \frac{1}{2}\left[\left(r-\mu^{\prime}(x)\right)\left(R_{r} f\right)_{2}^{\prime}(x)-\mu(x)\left(R_{r} f\right)_{2}^{\prime \prime}(x)-f^{\prime}(x)\right],
$$

the sign of which is indeterminate.

\section{Concluding Comments}

In this article we have analyzed a firms optimal risk adoption in an environment where the adoption means a switch from one stochastic flow representation into another. We have demonstrated that increased volatility needs not decelerate investment, as predicted by the standard literature on real options, once the underlying volatility of the state variable is made endogenous. Within such a context we have shown that the optimal adoption timing represents a trade-off between the real option effect and the Jensen's inequality effect (these effects may operate in different directions). More precisely, from the point of view of a decision maker endowed with a convex (concave) objective function increased post-adoption volatility tends to increase (decrease) the expected cumulative present value of the postadoption cash flow, which tends to decrease (increase) the option value of waiting, which, in turn, accelerates (decelerates) current investment.

In order to focus exclusively on the risk characteristics we have restricted this study to technology upgrading which switches the riskiness of the profit flow while keeping the drift constant. Within a more general approach one could analyze adoption equilibria within a framework where the upgrading of the technology switches both the volatility and the drift of the underlying stochastic process. Another potential extension would be to exploit our approach in order to draw conclusions with respect to the design of optimal risk management within firms.

\section{References}

[1] Abel, A. Optimal investment under uncertainty, 1983, American Economic Review, vol 73, $228-233$.

[2] Abel, A. The effects of uncertainty on investment and the expected long-run capital stock, 1984, Journal of Economic Dynamics and Control, vol 7, 39 - 53.

[3] Abel, A. A stochastic model of investment, marginal $q$ and the market value of the firm, 1985, International Economic Review, vol 26, 305 - 322.

[4] Alvarez, L. H. R. Solving Optimal Stopping Problems of Linear Diffusions by Applying Convolution Approximations, 2001, Mathematical Methods of Operations Research, vol. $53,89-99$.

[5] Alvarez, L. H. R. and Stenbacka, R. Adoption of Uncertain Multi-stage Technology Projects: A Real Options Approach, 2001, Journal of Mathematical Economics, vol. 35, 71-97.

[6] Borodin, A. and Salminen, P. Handbook on Brownian motion - Facts and formulae, 1996, Birkhauser, Basel. 
[7] Caballero, R. J. On the sign of the investment-uncertainty relationship, 1991, American Economic Review, vol 81, 279 - 288.

[8] Dixit, A. K. Entry and exit decisions under uncertainty, 1989, Journal of Political Economy, vol 97, 620-638.

[9] Dixit, A. K. Investment and Hysteresis, 1992, Journal of Economic Perspectives, vol 6, $107-132$.

[10] Dixit, A. K. and Pindyck, R. S. Investment under uncertainty, 1994, Princeton UP, Princeton.

[11] Grenadier, S. R. Information revelation through option exercise, 1999, The Review of Financial Studies, vol. 12, 95-129.

[12] Hartman, R. The effects of price and cost uncertainty on investment, 1972, Journal of Economic Theory, vol 5, 258 - 266.

[13] Hartman, R. Adjustment costs, price and wage uncertainty, and investment, 1973, Review of Economic Studies, vol 40, 259 - 267.

[14] Lee, J. and Shin, K. The role of variable input in the relationship between investment and uncertainty, 2000, American Economic Review, vol 90, 667-680.

[15] Mandl, P. Analytical treatment of one-dimensional Markov processes, 1968, Springer, Prague.

[16] Pindyck, R. S. Irreversible investment, capacity choice, and the value of the firm, 1988, American Economic Review, vol 78, 969 - 985.

[17] Pindyck, R. S. Irreversibility, uncertainty, and investment, 1991, Journal of Economic Literature, vol 29, 1110 - 1152.

[18] Pindyck, R. S. A note on competitive investment under uncertainty, 1993, American Economic Review, vol 83, 273-277.

[19] Protter, P. Stochastic integration and differential equations, 1990, Springer, New York. 